\title{
Broadening the List of Basal Cell Carcinoma Mimickers: Dermoscopic Features of Trichoadenoma
}

\author{
Riccardo Pampena ${ }^{1}$, Stefania Borsari ${ }^{1}$, Simonetta Piana ${ }^{2}$, Caterina Longo ${ }^{1,3}$
}

\begin{abstract}
1 Centro Oncologico ad Alta Tecnologia Diagnostica, Azienda Unità Sanitaria Locale - IRCCS di Reggio Emilia, Italy 2 Pathology Unit, Azienda Unità Sanitaria Locale - IRCCS di Reggio Emilia, Italy

3 Department of Dermatology, University of Modena and Reggio Emilia, Modena, Italy
\end{abstract}

Key words: trichoadenoma, basal cell carcinoma, adnexal tumors, dermoscopy

Citation: Pampena R, Borsari S, Piana S, Longo C. Broadening the list of basal cell carcinoma mimickers: dermoscopic features of trichoadenoma. Dermatol Pract Concept. 2019;9(2):160-161. DOI: https://doi.org/10.5826/dpc.0902a17

Accepted: January 10, 2019; Published: April 30, 2019

Copyright: @2019 Pampena et al. This is an open-access article distributed under the terms of the Creative Commons Attribution License, which permits unrestricted use, distribution, and reproduction in any medium, provided the original author and source are credited.

Funding: This research was supported by Italian Ministry of Health (Project Code: NET-2011-02347213).

Competing interests: The authors have no conflicts of interest to disclose.

Authorship: All authors have contributed significantly to this publication.

Corresponding author: Riccardo Pampena, MD, Centro Oncologico ad Alta Tecnologia Diagnostica, Azienda Unità Sanitaria Locale IRCCS, Viale Risorgimento 80, 42123, Reggio Emilia, Italy. Email: riccardopampena@gmail.com

\section{Introduction}

A wide spectrum of skin tumors may mimic basal cell carcinoma (BCC) on both clinical and dermoscopic appearance. Among these, adnexal skin neoplasms and in particular benign follicular tumors are commonly indistinguishable from BCC [1]. Benign follicular tumors are uncommon skin neoplasms, mainly represented by trichoblastoma and trichoepithelioma; however, other rarer subtypes may be also encountered, such as trichoadenoma, trichofolliculoma, and panfolliculoma. To the best of our knowledge, no reports have previously described the dermoscopic appearance of trichoadenoma. Herein we report a case of a man in his 40s who had a long-standing 5-mm papule located on his left eyebrow; the lesion was flesh-colored, firm, and painless (Figure1A).

\section{Case Presentation}

Dermoscopic evaluation was performed with a contact polarized dermatoscope (DermLite Foto, 3Gen LLC, Dana Point, CA, USA) and showed a general BCC-like appearance. In particular, crystalline structures could be identified through the lesion, together with an ovoid blue-gray area at the periphery of the tumor and diffuse on-focus linear vessels, visible only when gentle pressure was applied. In addition, another dermoscopic feature could also be observed that is not typically seen in BCC, consisting of small whitish circles with a diffuse distribution (Figure 1, B and C). The lesion was excised with the suspicion of BCC. The histopathological diagnosis was trichoadenoma (Figure 1D).

Trichoadenoma is an uncommon benign tumor originating from the infundibular part of the pilosebaceous unit, 
which was first described in 1958 by Nikolowski as "organoid follicular hamartoma." Generally, it involves adults without sex predilection and appears as a solitary skin-colored papule. More than half of cases have been described on the face and almost one quarter on the buttock [2]. Occasionally, trichoadenoma was also reported in association with a nevus sebaceous. Histopathologically, it shows a level of organization intermediate between trichofolliculoma and trichoepithelioma, with distinctive features from both of these neoplasms. In particular, trichoadenoma commonly appears as a well-circumscribed, dermal nodule, characterized by multiple infundibulocystic structures lined with stratified squamous epithelium, with a granular layer, showing epidermoid differentiation and resembling multiple cross-sections of the infundibular portion of hair follicles. However, hair shafts are generally absent. The main differential diagnosis of trichoadenoma is represented by desmoplastic trichoepithelioma, and some authors have suggested that trichoadenoma could even be a variant of the latter. However, clinical, histopathological, and immunohistochemical differences suggest that these tumors should be considered distinct entities [2].

\section{Conclusions}

In this report we first described the dermoscopic features of trichoadenoma, showing that this tumor should also be considered in the broad spectrum

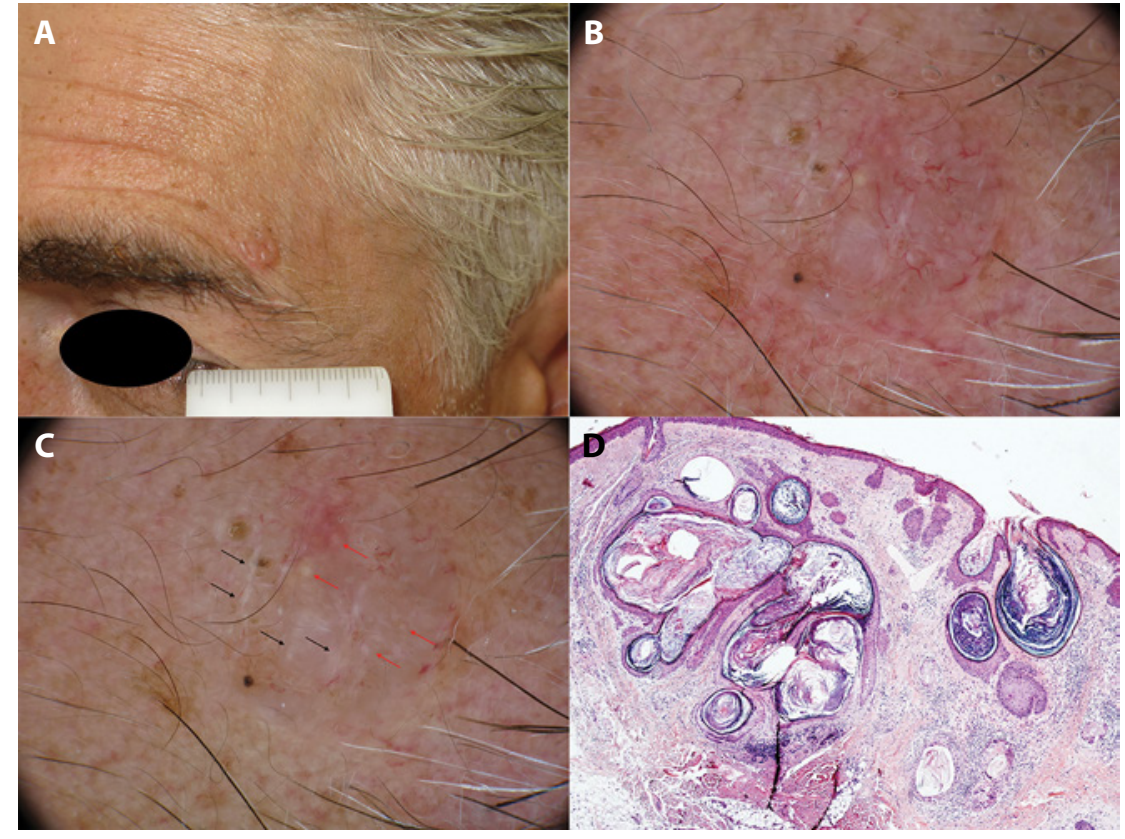

Figure 1. (A) Clinical picture revealing a 5-mm flesh-colored papule over the left eyebrow of a 45-year-old man. (B,C) Dermoscopic pictures with polarized light, without (B) and with (C) compression. On-focus linear vessels may be seen when compression was not applied; in addition, an ovoid blue-gray area was visible at the periphery of the tumor. When compression was not applied, white structures were more visible: in particular, shiny white lines (black arrows) and small whitish circles (red arrows). (D) At histological examination, the dermis showed irregularly shaped keratinous cysts, many filled with keratotic material and lined with stratified squamous epithelium with epidermoid differentiation. Some cysts were superficial and opened into the epidermis (hematoxylin and eosin, $\times 40$ ). [Copyright: $\odot 2019$ Pampena et al.]

of BCC mimickers. In particular, we found a prevalence of crystalline structures, arranged as shiny white lines and white circles. The former were previously described as characterizing both BCCs and skin adnexal tumors [1]; the latter were also previously reported in adnexal tumors, but are commonly absent in BCCs. White circles are probably histopathologically correlated with the infundibulocystic structures characterizing trichoadenoma, although fur- ther studies are needed to support this preliminary hypothesis.

\section{References}

1. Lallas A, Moscarella E, Argenziano G, et al. Dermoscopy of uncommon skin tumors. Australas J Dermatol. 2014;55(1):53-62.

2. Shimanovich I, Krahl D, Rose C. Trichoadenoma of Nikolowski is a distinct neoplasm within the spectrum of follicular tumors. J Am Acad Dermatol. 2010;62(2):277-283. 